\title{
White Dwarf Seismology at the Université de Montréal
}

\author{
G. Fontaine, P. Brassard, P. Bergeron, F. Wesemael \\ Département de Physique, Université de Montréal
}

Over the last several years, we have developed a comprehensive program aimed at better understanding the properties of pulsating DA white dwarfs (or ZZ Ceti stars). These stars are nonradial pulsators of the $g$-type, and their study can lead to inferences about their internal structure. For instance, the period spectrum of a white dwarf is most sensitive to its vertical chemical stratification, and one of the major goals of white dwarf seismology is to determine the thickness of the hydrogen layer that sits on top of a star. This can be done, in principle, by comparing in detail theoretical period spectra with the periods of the observed excited modes. Likewise, because the cooling rate of a white dwarf is very sensitive to the specific heat of its core material (and hence to its composition), it is possible to infer the core composition through measurements and interpretations of rates of period change in a pulsator.

In order to provide the needed theoretical framework within which to analyze observed period spectra and measurements of rates of period change, we have recently completed a thorough adiabatic survey of evolutionary models of white dwarf stars (Ap.J. Suppl., 72, 335; Ap. J. Suppl. 81, 747). In the process, we have developed new analytical and numerical tools, including an analytical model for the phenomenon of trapping of gravity-modes at a composition discontinuity in a star (Ap. J. Suppl. 80, 369 ), as well as a high-performance pulsation code based on finite-element techniques (Ap. J. Suppl. 80, 725). We believe that these results will be instrumental in reaping the most important benefits from white dwarf seismology. We have also developed a new technique (based on numerical simulations of the emergent energy distribution of a pulsating white dwarf) which allows us to exploit the information contained in the relative amplitudes of a pulsation mode and its harmonics in a Fourier spectrum. This is particularly important for interpreting the high signalto-noise ratio observations which we have started gathering at the Canada-FranceHawaii Telescope with the help of "LAPOUNE", the Montréal 3-channel photometer. This observational effort constitutes an integral part of our white dwarf seismological program, and has produced, so far, data of unprecedented quality. In the immediate future, we expect that our observations coupled to our adiabatic survey data will allow us to infer the amount of hydrogen floating on top of several DA white dwarfs. 